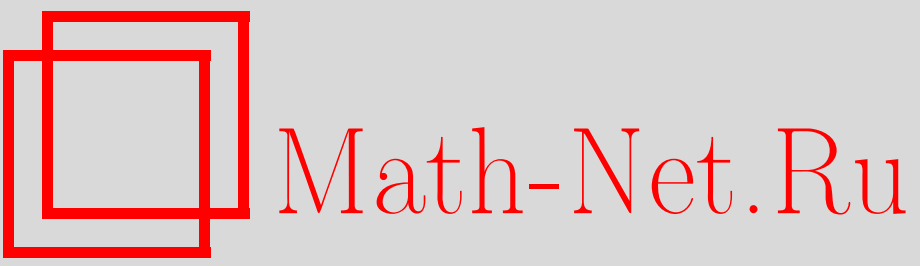

В. Т. Березин, Геометрическая структура базы и скрученность градуированных расслоений, используемых в моделировании гравитации и элементарных частиц, ТМФ, 1996, том 108, номер 1, 16-35

DOI: https://doi.org/10.4213/tmf1174

Использование Общероссийского математического портала Math-Net.Ru подразумевает, что вы прочитали и согласны с пользовательским соглашением

http://www.mathnet.ru/rus/agreement

Параметры загрузки:

IP : 18.208 .226 .222

26 апреля 2023 г., 15:56:38 
ТЕОРЕТИЧЕСКАЯ

И МАТЕМАТИЧЕСКАЯ

ФИЗИКА

Том 108, № 1

июль, 1996

В. Т. Березин

\section{ГЕОМЕТРИЧЕСКАЯ СТРУКТУРА БАЗЫ И СКРУЧЕННОСТЬ ГРАДУИРОВАННЫХ РАССЛОЕНИЙ, ИСПОЛЬЗУЕМЫХ В МОДЕЛИРОВАНИИ ГРАВИТАЦИИ И ЭЛЕМЕНТАРНЫХ ЧАСТИЦ}

\footnotetext{
В проблемах объединения фундаментальных взаимодействий элементарных частиц и гравитации возникает необходимость в привлечении математической структуры градуированного расслоения $\zeta$. Его базой $B$ служит 9 -мерное градуированное пространство, имеющее одно скалярное, 4 спинорных и 4 векторных измерения (относительно группы Лоренца). Найдено однопараметрическое семейство градуированных групп Пуанкаре $1 P$. Показано, что любая группа из этого семейства действует на своей инвариантной подгруппе и на базе $B$ по-разному. Эта ситуация отличается от классической и указывает на нетривиальность геометрических свойств расслоения $\zeta$. Обсуждена проблема скрученности расслоений.
}

\section{1. ВВЕДЕНИЕ}

Современные достижения в области физики элементарных частиц и гравитации - в эксперименте и теории - дают основание полагать, что реальное пространство-время имеет гораздо более сложную структуру за счет суперсимметрии [1-6]. Мы будем называть его базовым физическим пространством $B$, поскольку оно выступает всегда в

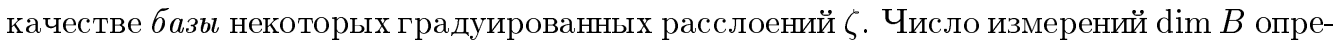
деляется расширенной группой градуированных преобразований $N P, N=1,2,3, \ldots$. Мы ограничимся случаем $N=1$, который охватывает всю совокупность современных экспериментальных данных по элементарным частицам. Группа $1 P$ минимальна с точки зрения числа входяших в нее грассман-нечетных параметров, но в то же время максимальна с точки зрения числа генераторов, образуюших единственную неприводимую градуированную алгебру Ли симметрий $S$-матрицы, которая возможна в рамках ковариантной квантовой теории поля (теорема Хаага, Лопушанского и Сониуса [7]). При $N=1$ имеем $\operatorname{dim} B=9$. Сейчас становится все более ясно, что важнейшими математическими структурами, действуюшими в физике элементарных частиц и гравитации, являются групповая и грассманова - из алгебраических структур, многообразия и расслоения - из геометрических. В своих исследованиях, начатых в [8], мы обнаружили, что с этой точки зрения большое значение имеет геометрический формализм градуиро- 
ванного расслоенного пространства со структурной группой

$$
G=G L(B) \times L \times G_{\mathrm{int}}, \quad G_{\mathrm{int}}=U(1) \times S U(2) \times S U(3) .
$$

Здесь $G_{\text {int }}$ - известная группа внутренних симметрий элементарных частиц, $L$ - группа Лоренца, $G L(B)$ - группа, действующая в пространствах касательных и кокасательных тензоров над базой $B$. Группа $G L(B)$ индуцируется янг-миллсовой группой $1 P_{\mathrm{Y}-\mathrm{M}}$, производящей для которой является $1 P$, при условии, что известно фундаментальное представление последней. В настояшей работе мы покажем, что сушествует однопараметрическое семейство генераторов этого представления (или, что равносильно, семейство фундаментальных представлений). Найден их явный вид.

В любой теории расслоения сушествует проблема скрученности, суть которой объясняется в разделе 2. Решение этой проблемы косвенно касается специфики вложения гравитации в теорию элементарных частиц. Однако эта тема выходит за рамки настоящей статьи.

Заметим, что вместо приставки "супер-" в таких словах, как супергруппа, мы используем прилагательное градуированныи. Это связано прежде всего с тем, что фундаментальные градуированные физические поля, преобразуюшиеся по представлениям группы (1), не реализуют никакого представления супергруппы $1 P$. В частности, киральные суперполя [1-3] нам не нужны. Соответственно стандартная суперсимметрия будет использована для весьма ограниченных целей. Кроме того, понятие “градуированныц им имеет четкий универсальный смысл, широко распространено в различных областях математики и общепринято.

\section{2. ПРОБЛЕМА ЗАДАНИЯ СКРУЧЕННОСТИ ГРАДУИРОВАННОГО РАССЛОЕНИЯ}

С помошью алгебры Грассмана обычное (классическое) расслоение [9-12] легко обобшается до градуированного. Пусть $A$-некоторая алгебра, $A_{\mathrm{ev}} \subset A, A_{\mathrm{od}} \subset A$, $\varnothing-$ пустое множество.

ОПредЕлЕниЕ 1 . Четностью в алгебре $A$ называется тройка

$$
\mathcal{P}=\left\{A_{\mathrm{ev}}, A_{\mathrm{od}}, \varphi \mid A_{\mathrm{od}} \neq \varnothing, \varphi: A_{\mathrm{ev}} \cup A_{\mathrm{od}} \rightarrow\{0,1\}\right\},
$$

удовлетворяюшая условиям:

а) $A_{\mathrm{ev}} \cap A_{\mathrm{od}}=\varnothing$;

б) элементы из $A_{\mathrm{ev}} \cup A_{\mathrm{od}}$ - образуюшие алгебры $A$;

в) ограничения $\varphi_{\mathrm{ev}}=\left.\varphi\right|_{A_{\mathrm{ev}}}, \varphi_{\mathrm{od}}=\left.\varphi\right|_{A_{\mathrm{od}}}$ постоянны, причем $\varphi_{\mathrm{ev}}: A_{\mathrm{ev}} \rightarrow 0$, $\varphi_{\text {od }}: A_{\text {od }} \rightarrow 1$;

г) для любых элементов $u, v \in A_{\mathrm{ev}} \cup A_{\mathrm{od}}$ выполняется равенство $u v=(-1)^{\varphi(u) \varphi(v)} v u$.

Элементы $u \in A_{\mathrm{ev}}$ называются четными (или бозе-элементами), $v \in A_{\mathrm{od}}$ - нечетным.ми (или ферми-элементами). Нечетные элементы антикоммутируют. Равенство из пункта "Г" часто пишут проше: $u v=(-1)^{u v} v u$ или

$$
u^{i} v^{k}=(-1)^{i k} v^{k} u^{i}
$$


при наличии индексов, которые, таким образом, градуируются. Отображение $\varphi$ распространяется на всю алгебру $A$ по линейности. Формула (3) в частном случае тензора $M^{i k}=v^{i} u^{k}$ индуцирует обшее определение градуированного транспонирования (что отмечается индексом gt) произвольной матрицы $M^{i k}$ :

$$
\left(M^{\mathrm{gt}}\right)^{i k}=(-1)^{i k} M^{i k}
$$

Заметим, что в литературе $[13,14]$ можно встретить другие определения градуированного транспонирования, полезность которых определяется теми или иными целями.

ОПреДЕЛЕниЕ 2. Алгебра $A=A G$, для которой существует (или задана) четность $\mathcal{P}$, назьвается алгеброй Грассмана, а геометрическая структура над ней называется градуированной.

По алгебре Грассмана имеется обширная литература, например $[4,13,14]$. Градуированное $G$-расслоение с тотальным пространством $E$, базой $B$, проекцией $\pi$ (т.е. сюръективным отображением $\pi: E \rightarrow B)$, типичным слоем $F$, атласом расслоения $\Phi$ и структурной группой $G$ из (1) будем обозначать символом $\zeta=(E, \pi, B, F, \Phi, G)$. Атлас есть набор пар $\left(U_{A}, \chi_{A}\right), A \in \mathcal{A}$, где $\left\{U_{A}\right\}$ - открытое покрытие $B, \chi_{A}: U_{A} \times$ $F \rightarrow \pi^{-1} U_{A} \subset E$ - так называемые тривиализирующие гомеоморфизмы или локальные структурь прямого произведения.

Пусть $\rho: E \times G \rightarrow E$ - действие $G$ на $E$ справа, $\lambda: G \times F \rightarrow F$ - действие $G$ на $F$ слева. Для любых $A, A^{\prime} \in \mathcal{A}, b \in U_{A} \cap U_{A^{\prime}}, v \in F$ формулой

$$
g_{A A^{\prime}}(b) \cdot v=\chi_{A}^{-1} \circ \chi_{A^{\prime}}(b, v),
$$

где точка $(\cdot)$ - действие $\lambda$, задаются функиии перехода $g_{A A^{\prime}}: U_{A} \cap U_{A^{\prime}} \rightarrow G$. Они имеют свойства

$$
\begin{aligned}
g_{A A^{\prime}}(b) g_{A^{\prime} A^{\prime \prime}}(b) & =g_{A A^{\prime \prime}}(b), \quad b \in U_{A} \cap U_{A^{\prime}} \cap U_{A^{\prime \prime}}, \\
g_{A A^{\prime}}(b) & =g_{A^{\prime} A^{-1}}^{-1}(b), \\
g_{A A}(b) & =e \quad(e \in G-\text { единичный элемент) }
\end{aligned}
$$

и задают скручивание между двумя пересекающимися локальными структурами прямого произведения. Это означает, что диаграмма

$$
\begin{array}{ccccc}
U_{A^{\prime}} \times F \supset\left(U_{A} \cap U_{A^{\prime}}\right) \times F \stackrel{\chi_{A}^{-1} \circ \chi_{A^{\prime}}}{\longrightarrow}\left(U_{A} \cap U_{A^{\prime}}\right) \times F \subset U_{A} \times F \\
\downarrow \chi_{A^{\prime}} & \chi_{A^{\prime}} \searrow & \swarrow \chi_{A} & \downarrow \chi_{A} \\
\pi^{-1} U_{A^{\prime}} & \supset & \pi^{-1}\left(U_{A} \cap U_{A^{\prime}}\right) & \subset & \pi^{-1} U_{A}
\end{array}
$$

коммутативна. Таким образом, пространство $E$ локально является прямым произведением, а в целом скручено. Пример - лист Мебиуса.

Если $F=V$ - векторное пространство с послойно линейными тривиализирующими гомеоморфизмами, то градуированное $G$-расслоение называется векторным. Если же $F=G$ с правым групповым действием $\chi_{A}(b, g)=\chi_{A}(b, e) \cdot g$, то $\zeta$ называется главным градуированным $G$-расслоением. Здесь точка означает действие $\rho, b \in U_{A}$, $g \in G, e \in G$ - единичный элемент. Градуированное сечение $\sigma: B \rightarrow E, \pi \circ \sigma=\mathrm{id}_{B}$, 
сопоставляет каждому $b \in B$ некоторый элемент слоя $\pi^{-1}(b) \subset E$. Сечения $\sigma_{1}$ и $\sigma_{2}$ главного $G$-расслоения можно послойно перемножать в групповом смысле: $\left(\sigma_{1}\right.$. $\left.\sigma_{2}\right)(b)=\sigma_{1}(b) \cdot \sigma_{2}(b)$. Множество $\{\sigma\}$ сечений главного $G$-расслоения называется гр $a d y$ -

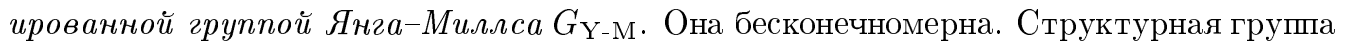
$G$ называется производящей для $G_{\mathrm{Y}-\mathrm{M}}$. Функции перехода $g_{A A^{\prime}}(b)$ суть некоторые сечения главных подрасслоений над $U_{A} \cap U_{A^{\prime}}$ для всех $A, A^{\prime} \in \mathcal{A}$.

В индуцированной естественным образом $G$-расслоениями $\zeta$ модели элементарных частиц и гравитации (для простоты будем ее называть bundle model или короче $B$-моделью) наряду с главным $G$-расслоением и его различными сечениями используются векторные $G$-расслоения, типичные слои которых являются пространствами неприводимых представлений группы (1) с индексацией прямого произведения. В обоих случаях базой служит некоторое многообразие $B$, вообще говоря, с неизвестной топологией $[8]$. В принципе $B$ может быть многосвязным с различными физическими и даже размерными свойствами в различных компонентах связности и выполняет функции пространства-времени. Однако эти функции расширены за счет градуировки. Все фермионные $G$-расслоения скрученные в смысле диаграммы (7), о чем свидетельствует наличие спина у частиц. Это обстоятельство порождает проблему. Дело в том, что для произвольных $G$-расслоений и в математике, и в физике отсутствуют естественные (канонические) способы задания тривиализируюших отображений $\chi_{A}$ и, следовательно, функций перехода $g_{A A^{\prime}}(b)$. Их задание составляет важное звено в аксиоматизации теории, что будет рассмотрено в разделе 7. Знание функций перехода необходимо для общековариантной формулировки теории, в частности ковариантного описания спина, корректного включения гравитации в теорию элементарных частиц и выполнения обшего трансформационного анализа [8]. В решении указанной проблемы большую роль играет принцип соответствия, обеспечивающий предельный переход к стандартной модели Глэшоу-Вайнберга-Салама [15-17].

\section{3. ЗАДАНИЕ ГРАДУИРОВАННОЙ ГРУППЫ ПУАНКАРЕ $1 P$, БАЗЫ $В$ И ИХ ПАРАМЕТРИЗАЦИЯ}

Пусть заданы линейное пространство $F_{\mathbb{M}}=\mathbb{M}\left(n_{\mathbb{M}}, A G\right)$ матриц размера $n_{\mathbb{M}} \times n_{\mathbb{M}} \mathrm{c}$ элементами, возможно, из алгебры Грассмана $A G$ и тривиальное расслоение

$$
\zeta_{\mathbb{M}}=\left(E_{\mathbb{M}}=B \times F_{\mathbb{M}}, \pi_{\mathbb{M}}, B, F_{\mathbb{M}}, \Phi_{\mathbb{M}}, G=e\right)
$$

с проекцией $\pi_{\mathbb{M}}$ на первый сомножитель прямого произведения, типичным слоем $F_{\mathbb{M}}$ и тождественной структурной группой $G$ (т.е. состояшей из единичного элемента $e$ ). Матричные блоки и сечения расслоения с четными и нечетными элементами называются соответственно четными и нечетными. Будем использовать следуюшую индексацию: для аналогов пространства Минковского $i, j, k, l, \ldots=0,1,2,3$ (строчные буквы из середины алфавита), для аналогов пространства спинорного представления $a, b, c, \ldots=$ $1,2,3,4$ (буквы из начала алфавита), для наборов индексов $M, N, \ldots=(m, a, \varnothing)$ (прописные буквы из середины алфавита). Подъем и опускание индексов типа $i, j$ осушествляется с помощью матрицы

$$
\eta_{v}=\left\|\eta_{m n}\right\|=\operatorname{diag}(-1,1,1,1)=\left\|\eta^{m n}\right\| .
$$


Пусть $\gamma^{m}=\left\|\left(\gamma^{m}\right)_{b}^{a}\right\|$ - матрицы Дирака, где $a$ - номер строки, $b$ - номер столбца, $\Sigma^{m n}=\frac{1}{4}\left(\gamma^{m} \gamma^{n}-\gamma^{n} \gamma^{m}\right)-$ генераторы спинорного представления группы Лоренца, $\gamma_{5}=-i \gamma^{0} \gamma^{1} \gamma^{2} \gamma^{3}$. Антисимметричная матрица $C=\left\|C_{a b}\right\|$ зарядового сопряжения инвариантна относительно спинорного преобразования. Поэтому $C$ или $C^{-1}=\| C^{a b \|}$ удобно считать метрическим тензором в аналогах пространства спинорного представления. Это означает, что с помошью $C^{-1}$ можно поднимать спинорные индексы, а с помошью $C$ - опускать. Как обычно, [, ] - коммутатор, $\{$,$\} - антикоммутатор (любой из$ них в градуированной символике обозначается как $[\}$,$) , по повторяющимся индексам$ всех сортов подразумевается суммирование.

ТЕОРема 1. Если существуют такое челое число $n_{\mathbb{M}}>0$ и такие 15 сечений

$$
\sigma_{\mathcal{M}} \in\left\{\sigma_{M}, J_{m n}=-J_{n m}\right\}, \quad \sigma_{M}=\left\{P_{m}, Q_{a}, R=R_{\varnothing}\right\}
$$

расслоения (8), которье удовлетворяют коммутационным соотношениям [1-3]

$$
\begin{aligned}
{\left[P_{m}, P_{n}\right] } & =\left[Q_{a}, P_{m}\right]=\left[P_{m}, R\right]=\left[J_{m n}, R\right]=0, \\
{\left[P_{m}, J_{n l}\right] } & =\eta_{m n} P_{l}-\eta_{m l} P_{n}, \\
{\left[J_{m n}, J_{k l}\right] } & =\eta_{m l} J_{n k}+\eta_{n k} J_{m l}-\eta_{m k} J_{n l}-\eta_{n l} J_{m k}, \\
\left\{Q_{a}, Q_{b}\right\} & =2\left(\gamma_{m} C\right)_{a b} P^{m} \\
{\left[Q_{a}, J_{m n}\right] } & =-\left(\Sigma_{m n}\right)_{a}^{b} Q_{b}, \\
{\left[Q_{a}, R\right] } & =i\left(\gamma_{5}\right)_{a}^{b} Q_{b},
\end{aligned}
$$

то $\sigma_{\mathcal{M}}$ образуют замкнутую градуированную (т.е. с существующей четностью) алгебру Ли $1 p$.

ДокАЗАТЕЛЬСтво. Нужно проверить градуированные тождества Якоби

$$
\left[\left[\sigma_{\mathcal{M}}, \sigma_{\mathcal{N}}\right\}, \sigma_{\mathcal{L}}\right\}+\left[\left[\sigma_{\mathcal{N}}, \sigma_{\mathcal{L}}\right\}, \sigma_{\mathcal{M}}\right\}+\left[\left[\sigma_{\mathcal{L}}, \sigma_{\mathcal{M}}\right\}, \sigma_{\mathcal{N}}\right\}=0
$$

и убедиться, что они выполняются [1-3]

В (11) $Q_{a}$ - нечетные матрицы, остальные четные. Таким образом, сечения $\sigma_{\mathcal{M}}-$ генераторы градуированной группы Пуанкаре $1 P$. Решение вопроса о сушествовании сечений $\sigma_{\mathcal{M}}$ отложим до раздела 5 (теорема 2). Можно показать $[1,2]$, что для четырех нечетных генераторов $Q_{a}$, образуюших майоранов спинор со значениями компонент в $\mathbb{M}\left(n_{\mathbb{M}}, A G\right)$, группа $1 P$ максимальна, т.е. она не допускает расширения. С другой стороны, $1 P$ не может иметь меньше четырех ненулевых генераторов $Q_{a}$. Это важно с точки зрения сушествования и единственности теоретических построений в физике. Соотношения (11) обладают лоренцевой симметрией, если предположить, что $R$ - скаляр, $Q_{a}$ спинор. Легко видеть, что эта симметрия продолжается до янг-миллсовой симметрии, когда преобразования Лоренца зависят от точек $b \in B$ базы расслоения (8). Поэтому в принципе нет необходимости требовать постоянства сечений $(10)$, хотя над любой координатной окрестностью базы $B$ эти сечения могут быть постоянными. Возможность же глобального постоянства сечений $(10)$ зависит от топологии пространства $B$.

Группу $1 P$ можно параметризовать либо с помощью формулы

$$
g(\bar{a}, \bar{\omega})=\exp \left(\bar{a}^{m} P_{m}+\bar{\varepsilon}^{a} Q_{a}+\bar{\rho} R+\frac{1}{2} \bar{\omega}^{m n} J_{m n}\right) \in 1 P,
$$


где $\bar{a}=\left(\bar{a}^{m}, \bar{\varepsilon}^{a}, \bar{\rho}=\bar{\rho}^{\varnothing}\right)=\left\{\bar{a}^{M}\right\}$, либо с помошью формулы

$$
g(a, \omega)=\exp \left(a^{m} P_{m}+\varepsilon^{a} Q_{a}+\rho R\right) \exp \left(\frac{1}{2} \omega^{m n} J_{m n}\right) \in 1 P
$$

либо как-то иначе. Разные параметризации отвечают разным системам координат в группе $1 P$, рассматриваемой как многообразие. Из (11) видно, что генераторы $\sigma_{M}$ образуют идеал $1 t$ в $1 p$, так что

$$
1 T=\left\{\exp \left(a^{m} P_{m}+\varepsilon^{a} Q_{a}+\rho R\right)\right\}
$$

- нормальная (инвариантная) подгруппа в $1 P$. Тогда подгруппу

$$
L=\left\{\exp \left(\frac{1}{2} \omega^{m n} J_{m n}\right)\right\} \subset 1 P
$$

являюшуюся, вообще говоря, представлением группы Лоренца, можно рассматривать как фактор-группу $L=1 P / 1 T$ по модулю $1 T$ при условии, что отношение эквивалентности $g_{1} \sim g_{2}$ элементов $1 P$ определено с помощью правых смежных классов (при другой параметризации значение имели бы левые смежные классы).

В идеале $1 t=\left\{a^{m} P_{m}+\varepsilon^{a} Q_{a}+\rho R\right\} \subset 1 p$ параметры $a^{m}$ и $\rho$ четны, $\varepsilon^{a}$ нечетны. Тем не менее идеал $1 t$, как и вся градуированная алгебра Ли $1 p,-$ множество четных матриц размера $n_{\mathbb{M}} \times n_{\mathbb{M}}$. Предполагается, что $\varepsilon^{a} Q_{a}-$ свертка майорановых спиноров.

Каждому элементу $g_{1 t} \in 1 t$ может быть сопоставлена точка $z=\left(a^{m}, \varepsilon^{a}, \rho=\rho^{\varnothing}\right)$ некоторого линейного 9 -мерного пространства $B_{c}$. В качестве аксиомы примем утверждение, что $B_{c}$ совпадает с подстилающим (координатным, - отсюда индекс $c$ ) пространством базы $B$ в расслоении (8), необходимом для задания тривиализирующих отображений многообразия $B$ в линейное пространство с целью введения систем координат. В эту аксиому включили также предположение о том, что $B$ - физическая модель реального пространства-времени с некоторой топологией, а $B_{c}$ - девятимерное градуированное пространство с метрикой

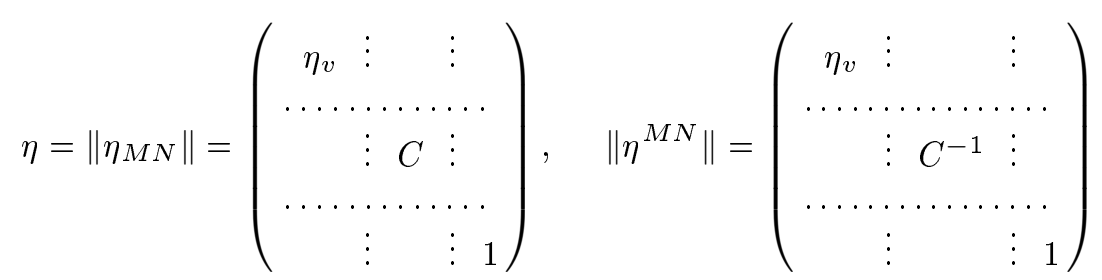

(незаполненные блоки нулевые, $C$ - матрица зарядового сопряжения). Будем называть $B$ базовым физическим пространством, $B_{c}-$ координатным физическим пространством. Вопрос об их топологической эквивалентности остается открытым. Доказательство того, что (17) - метрика, см. в разделе 6.

Известно, что классическая группа Пуанкаре $P$ действует одинаково и на инвариантной подгруппе $T=\left\{\exp \left(a^{m} P_{m}\right)\right\}$, и на пространстве Минковского $M^{4}$. Мы покажем, что для градуированных групп $1 P, 1 T$ и пространства $B_{c}$ это свойство уже не выполняется. 


\section{4. ИНФИНИТЕЗИМАЛЬНОЕ ДЕЙСТВИЕ ГРУППЫ $1 P$ НА ИНВАРИАНТНОЙ ПОДГРУППЕ $1 T$}

Выберем групповые координаты по схеме (14). Пусть

$$
g(z)=\exp \left(z^{N} \sigma_{N}\right) \in 1 T, \quad z=\left(z^{N}\right)=\left(x^{m}, \theta^{a}, r\right),
$$

а элемент (14) близок к единичному, т.е. $a=\left(a^{m}, \varepsilon^{a}, \rho\right)$ и $\omega=\left(\omega^{m n}\right)$ инфинитезимальны. Тогда, например, левое инфинитезимальное действие группы $1 P$ на подгруппе $1 T$ определяется формулой

$$
\begin{aligned}
g(a, \omega) g(z) & =\exp \left(a^{M} \sigma_{M}\right) \exp \left(\frac{1}{2} \omega^{m n} J_{m n}\right) \exp \left(z^{N} \sigma_{N}\right)= \\
& =\exp \left(z^{\prime N} \sigma_{N}\right) \exp \left(\frac{1}{2} \omega^{m n} J_{m n}\right)
\end{aligned}
$$

где $z^{\prime}=z+\xi$, причем $\xi^{M}\left(a^{m}, \varepsilon^{a}, \rho, \omega^{m n}\right)$ - некоторый сдвиг координат в пространстве параметров подгруппы $1 T$. Перепишем (18) в виде

$$
e^{X} e^{\frac{1}{2} \omega^{m n} J_{m n}} e^{z^{N} \sigma_{N}} e^{-\frac{1}{2} \omega^{k l} J_{k e}}=e^{z^{\prime N} \sigma_{N}}
$$

где $X=a^{M} \sigma_{M}$, и воспользуемся общей формулой

$$
e^{a A} B e^{-a A}=\sum_{s=0}^{\infty} \frac{a^{s}}{s !}(\operatorname{ad} A)^{s} B
$$

$(\operatorname{ad} A) B \equiv[A, B]$. Тогда в линейном приближении по $\omega^{m n}$ находим

$$
\begin{aligned}
& e^{\frac{1}{2} \omega^{m n} J_{m n}} e^{z^{N} \sigma_{N}} e^{-\frac{1}{2} \omega^{k l} J_{k l}}= \\
& \quad=\exp \left(e^{\frac{1}{2} \omega^{m n} J_{m n}} z^{N} \sigma_{N} e^{-\frac{1}{2} \omega^{k l} J_{k l}}\right) \simeq \\
& \quad \simeq \exp \left(z^{N} \sigma_{N}+\left[\frac{1}{2} \omega^{m n} J_{m n}, z^{N} \sigma_{N}\right]\right)=e^{Y}
\end{aligned}
$$

где

$$
Y=\left(x^{m}+\omega^{m n} x_{n}\right) P_{m}+\theta^{a}\left(1+\frac{1}{2} \omega^{m n} \Sigma_{m n}\right)_{a}^{b} Q_{b}+r R .
$$

После подстановки (21) в (19) применим формулу обшего вида

$$
e^{X} e^{Y}=\exp \left[Y+\sum_{s=0}^{\infty} \frac{B_{s}}{s !}(\operatorname{ad} Y)^{s} X+\cdots\right]
$$

(см. [18, с. 131]), где $X$ считается малой матрицей, $B_{s}$ - числа Бернулли. Напомним, что $B_{0}=1, B_{1}=-\frac{1}{2}, B_{2}=\frac{1}{6}, \ldots$ С помощью градуированных коммутаций (11) находим

$$
\sum_{s=0}^{\infty} \frac{B_{s}}{s !}(\operatorname{ad} Y)^{s} X=\left(a^{m}+a_{1}^{m}\right) P_{m}+\left(\varepsilon^{a}+\varepsilon_{1}^{a}\right) Q_{a}+\rho R .
$$


Здесь введены вспомогательные функциональные (зависящие от $\theta^{a}$ и $r$ ) параметры

$$
\begin{aligned}
a_{1}^{m} & =\left[B_{1} \varepsilon+(\rho \theta-\varepsilon r) i \gamma_{5} \sum_{s=2}^{\infty} \frac{B_{s}}{s !}\left(-i r \gamma_{5}\right)^{s-2}\right]^{a}\left(2 \theta \gamma^{m} C\right)_{a} \\
\varepsilon_{1}^{a} & =\left[(\rho \theta-\varepsilon r) i \gamma_{5} \sum_{s=1}^{\infty} \frac{B_{s}}{s !}\left(-i r \gamma_{5}\right)^{s-1}\right]^{a} .
\end{aligned}
$$

Всюду, где не указано явно, свертка по спинорным индексам выполняется в “северо-западном" направлении [1, приложение А]. Например,

$$
\left[(\rho \theta-\varepsilon r) i \gamma_{5}\right]^{a}\left(\theta \gamma^{m} C\right)_{a}=\left(\rho \theta^{b}-\varepsilon^{b} r\right) i\left(\gamma_{5}\right)_{b}^{a} \theta^{c}\left(\gamma^{m}\right)_{c}^{d} C_{d a}
$$

В итоге соотношение (19) принимает вид

$$
\exp (Y+\text { правая часть }(24))=\exp \left(z^{\prime N} \sigma_{N}\right)
$$

Отсюда получаем следующие формулы инфинитезимальных преобразований групповых градуированных координат:

$$
\begin{aligned}
x^{\prime m} & =x^{m}+\omega^{m n} x_{n}+a^{m}+a_{1}^{m}, \\
\theta^{\prime a} & =\theta^{a}+\frac{1}{2} \omega^{m n}\left(\theta \Sigma_{m n}\right)^{a}+\varepsilon^{a}+\varepsilon_{1}^{a}, \\
r^{\prime} & =r+\rho .
\end{aligned}
$$

В случае параметризации (13) исходя из формулы

$$
\begin{aligned}
g(\bar{a}, \bar{\omega}) g(z) & =\exp \left(\bar{a}^{M} \sigma_{M}+\frac{1}{2} \bar{\omega}^{m n} J_{m n}\right) \exp \left(z^{N} \sigma_{N}\right)= \\
& =\exp \left(z^{\prime N} \sigma_{N}+\frac{1}{2} \bar{\omega}^{\prime m n} J_{m n}\right)
\end{aligned}
$$

аналогичным путем находим преобразование

$$
\begin{aligned}
x^{\prime m} & =x^{m}+\frac{1}{2} \bar{\omega}^{m n} x_{n}+\bar{a}^{m}+\bar{a}_{1}^{m}+\bar{a}_{2}^{m}, \\
\theta^{\prime a} & =\theta^{a}+\varepsilon^{a}+\bar{\varepsilon}_{1}^{a}+\bar{\varepsilon}_{2}^{a}, \\
r^{\prime} & =r+\bar{\rho}, \quad \bar{\omega}^{\prime m n}=\bar{\omega}^{m n} .
\end{aligned}
$$

Здесь введены вспомогательные функции

$$
\begin{aligned}
\bar{a}_{2}^{m} & =-\frac{1}{2} \bar{\omega}^{k l}\left(\theta \Sigma_{k l}\right)^{a}\left(\theta \gamma^{m} C\right)_{a}, \\
\bar{\varepsilon}_{2}^{a} & =-\frac{1}{2} \bar{\omega}^{k l}\left[\theta \Sigma_{k l} \sum_{s=1}^{\infty} \frac{B_{s}}{s !}\left(-i r \gamma_{5}\right)^{s-1}\right]^{a},
\end{aligned}
$$


а функции $\bar{a}_{1}^{m}$ и $\bar{\varepsilon}_{1}^{a}$ имеют вид $(25)$ с заменой $\left(a^{M}\right) \rightarrow\left(\bar{a}^{M}\right)=\left(\bar{a}^{m}, \bar{\varepsilon}^{a}, \bar{\rho}\right)$. Если отождествить $z^{N}$ и $z^{\prime N}$ в (27) соответственно с $z^{N}$ и $z^{\prime N}$ в (29), то нетрудно получить преобразования $a^{M} \rightarrow \bar{a}^{M}, \omega^{m n} \rightarrow \bar{\omega}^{m n}$ групповых координат при переходе от параметризации (14) к параметризации (13). В частности, $\omega^{m n}=\frac{1}{2} \bar{\omega}^{m n}, \rho=\bar{\rho}$. Для остальных параметров формулы сложны.

В обоих случаях действия группы $1 P$ на подгруппе $1 T$ нелинейны по $\theta$ и $r$ и потому неудобны для теоретических исследований. Кроме того, генераторы этих действий, вычисленные по стандартной схеме дифференцирования в нуле матрицы преобразования по параметрам (ср. (32)-(34) ниже), не удовлетворяют соотношениям (11) и, следовательно, не реализуют линейного представления алгебры Ли $1 p$. Более того, они вообще перестают быть постоянными (на что хотелось бы рассчитывать), т.к. при вычислении их зависимость от $\theta$ и $r$ неустранима.

Все эти наблюдения говорят о том, что преобразования (27) или (29) (и, очевидно, любые другие с различным выбором групповых координат) не могут служить основой для построения фундаментального представления градуированной группы $1 P$, генераторы которого имели бы коммутационные свойства (11). Насколько нам известно, в литературе эту ситуацию, отличную от классической, никогда не принимают во внимание.

\section{5. ИНФИНИТЕЗИМАЛЬНОЕ ДЕЙСТВИЕ ГРУППЫ $1 P$ НА КООР ДИНАТНОМ ФИЗИЧЕСКОМ ПРОСТРАНСТВЕ}

Результаты предыдущего раздела свидетельствуют о том, что фундаментальное представление группы $1 P$ следует находить эвристически. Извлекая некоторую пользу из формул (27), будем искать инфинитезимальное действие группы $1 P$ на $B_{c}$ в виде

$$
\begin{aligned}
x^{\prime m}= & x^{m}+\omega^{m n} x_{n}+a^{m}+k_{1} \varepsilon^{a}\left(\theta \gamma^{m} C\right)_{a}, \\
\theta^{\prime a}= & \theta^{a}+\frac{1}{2} k_{2} \omega^{m n}\left(\theta \Sigma_{m n}\right)^{a}+k_{3} \varepsilon^{a}+ \\
& +\left[\left(k_{4} \rho \theta+k_{5} \varepsilon r\right) i \gamma_{5}\right]^{a}, \\
r^{\prime}= & +\rho .
\end{aligned}
$$

Здесь символика имеет прежнюю классификацию, но $z=\left(x^{m}, \theta^{a}, r\right)$ относится не к пространству параметров группы $1 T$, а к координатному пространству $B_{c}, z \in B_{c}$ (хотя это отличие несколько условно: оно сводится лишь к отличию преобразований). Числовые коэффищиенты $k_{i}, i=\overline{1,5}$, найдем из требования, чтобы генераторы преобразования (31) удовлетворяли соотношениям (11). Для этого записываем (31) в матричном виде

$$
\left(\begin{array}{c}
z^{\prime} \\
1
\end{array}\right)=\left(\begin{array}{cc}
\Lambda & \alpha \\
0 & 1
\end{array}\right) \cdot\left(\begin{array}{l}
z \\
1
\end{array}\right),
$$

т.e.

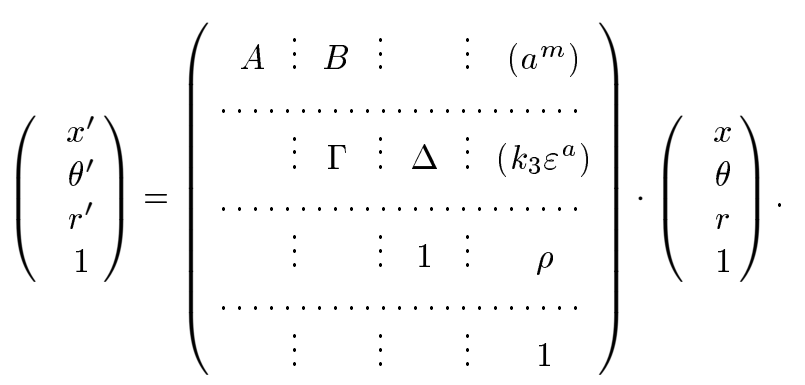


Здесь все незаполненные блоки нулевые. $(4 \times 4)$-матрицы $A, B, \Gamma$ и $(4 \times 1)$-столбец $\Delta$ имеют вид

$$
\left\{\begin{array}{l}
A=\operatorname{diag}(1,1,1,1)+\left\|\omega^{m n}\right\| \\
B=\left\|B_{a}^{m}\right\|=\left\|k_{1} \varepsilon^{b}\left(\gamma^{m}\right)_{a}^{c} C_{c b}\right\| \\
\Gamma=\left\|\Gamma_{b}^{a}\right\|=\left\|\delta_{b}^{a}+\frac{1}{2} k_{2} \omega^{m n}\left(\Sigma_{m n}\right)_{b}^{a}+k_{4} \rho\left(i \gamma_{5}\right)_{b}^{a}\right\| \\
\Delta=\left(\Delta^{a}\right)=\left(k_{5} \varepsilon^{b}\left(i \gamma_{5}\right)_{b}^{a}\right)
\end{array}\right.
$$

$(4 \times 1)$-столбцы $\left(a^{m}\right)$ и $\left(k_{3} \varepsilon^{a}\right)$ тривиальны. Вычисляя в групповой единице $e \in 1 P$ частные производные $(10 \times 10)$-матрицы из $(32)$ по групповым параметрам $\omega^{m n}, a^{m}, \varepsilon^{a}$ и $\rho$, получаем следующие $10 \times 10$-генераторы преобразования $(31)$ :

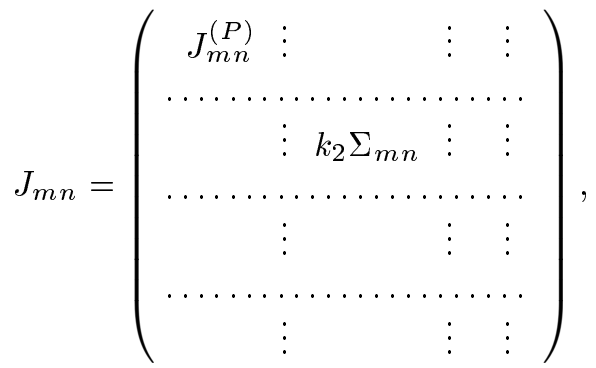

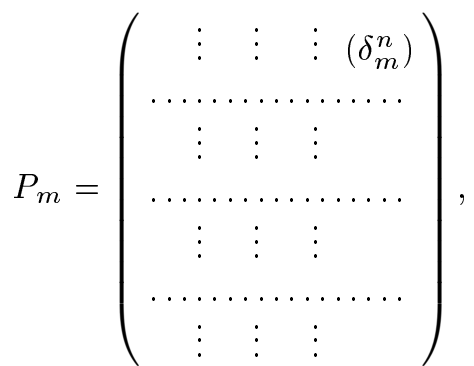

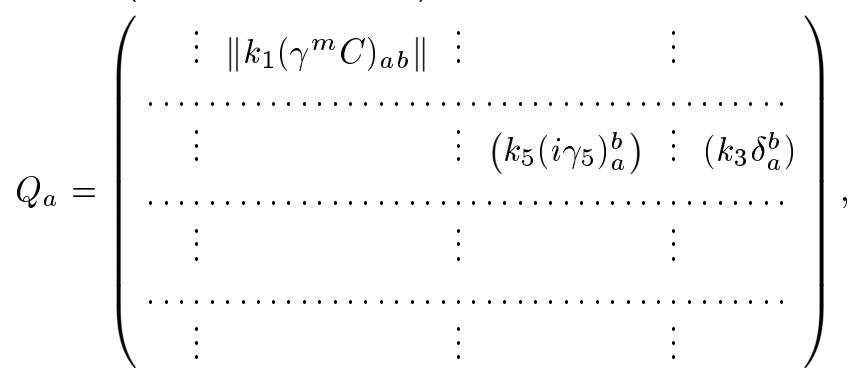

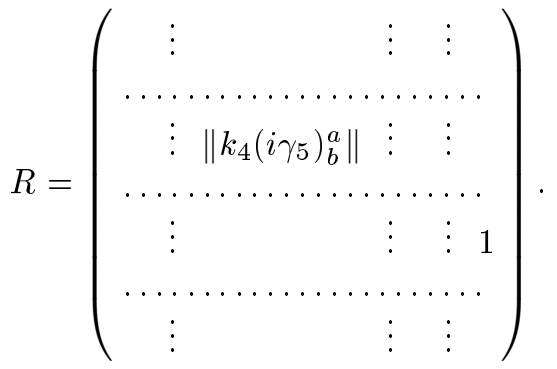


$J_{m n}^{(P)}$ - стандартньй врашательный генератор классической группы Пуанкаре $P$. Сразу замечаем, что в $J_{m n}$ должно быть $k_{2}=1$. Далее, находим, что $\left[Q_{a}, R\right]=\left(i \gamma_{5}\right)_{a}^{b} Q_{b}^{\prime}$, где

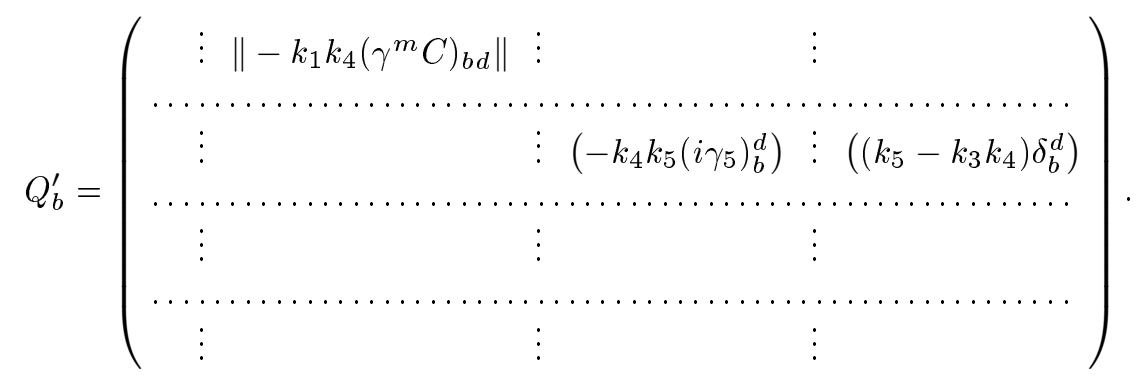

При получении этой матрицы было использовано свойство симметрии

$$
\left(\gamma^{m} C\right)_{a b}\left(i \gamma_{5}\right)_{d}^{b}=-\left(\gamma^{m} C\right)_{d b}\left(i \gamma_{5}\right)_{a}^{b}=-\left(\gamma^{m} C\right)_{b d}\left(i \gamma_{5}\right)_{a}^{b}
$$

Согласие вычисленного коммутатора с последним из (11) достигается только при условии, что $Q_{b}^{\prime}=Q_{b}$, т.е. если $k_{4}=-1, k_{5}=0$. Для антикоммутатора $\left\{Q_{a}, Q_{b}\right\}$ имеем $(\mathrm{c}$ учетом $k_{2}=-k_{4}=1, k_{5}=0$ )

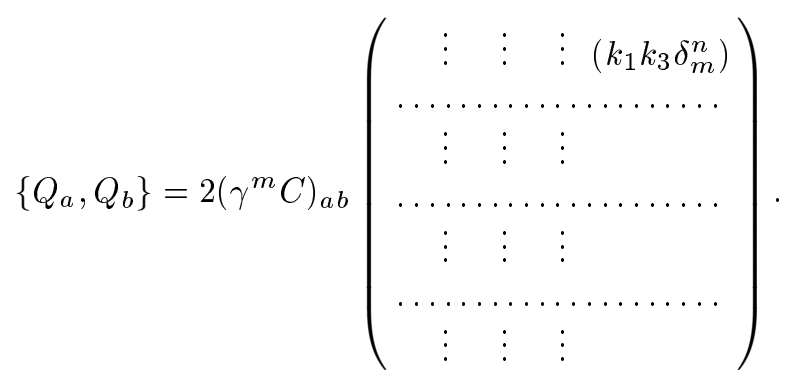

Из сравнения этого антикоммутатора с соответствующим из (11), а также с генератором $P_{m}$ из $(34)$ находим $k_{1} k_{3}=1$. Наконец, для коммутатора $\left[Q_{a}, J_{m n}\right]$ (по-прежнему с учетом $k_{2}=1, k_{5}=0$ ) получаем

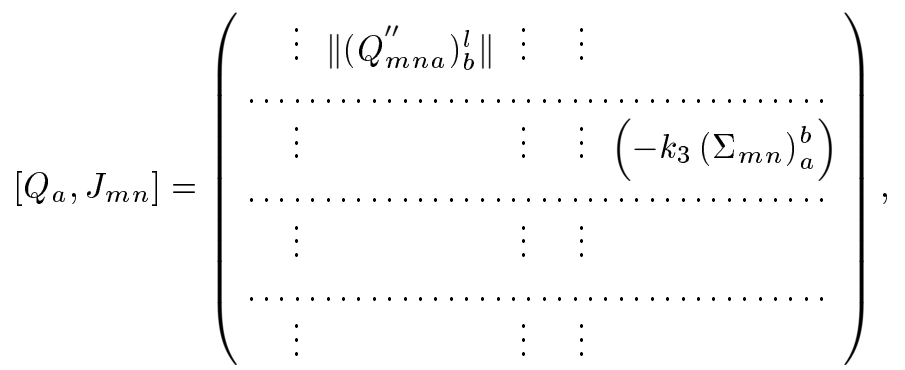

здесь

$$
\left\|\left(Q_{m n a}^{\prime \prime}\right)_{b}^{l}\right\|=\left\|k_{1}\left(\gamma^{l} C\right)_{a c}\left(\Sigma_{m n}\right)_{b}^{c}\right\|-k_{1}\left\|J_{m n}^{(P)}\right\| \cdot\left\|\left(\gamma^{l} C\right)_{a b}\right\| .
$$

Этот результат совпадает с предпоследним коммутатором в (11) при условии

$$
\left\|\left(Q_{m n a}^{\prime \prime}\right)_{b}^{l}\right\|=-\left(\Sigma_{m n}\right)_{a}^{d}\left\|k_{1}\left(\gamma^{l} C\right)_{d b}\right\|,
$$


где $\|\cdots\|$ - матрицы относительно индексов $l=\overline{0,3}$ и $b=\overline{1,4}$. Тогда получим $\left[Q_{a}, J_{m n}\right]=-\left(\Sigma_{m n}\right)_{a}^{b} Q_{b}$.

Условие (36) может быть записано в виде

$$
\left(\Sigma_{m n} \gamma^{l} C\right)_{a b}+\left(\Sigma_{m n} \gamma^{l} C\right)_{b a}=\left(J_{m n}^{(P)}\right)_{p}^{l}\left(\gamma^{p} C\right)_{a b}
$$

Лемма 1. Матрица $\Sigma_{m n} \gamma^{l}$ С относительно спинорных индексов антисимметрична для $l \neq m, n(m \neq n$; вариант $m=n$ тривиален $)$, симметрична при $l=m$ или $l=n$ и, кроме того, для всех $m, n, l=\overline{0,3}$ выполняется (37).

ДоКАЗАТЕЛЬСТВО. Исходим из известных соотношений

$$
\begin{aligned}
\left(J_{m n}^{(P)}\right)_{p}^{l} & =\eta_{m p} \delta_{n}^{l}-\eta_{n p} \delta_{m}^{l} \\
2 \Sigma^{m n} \gamma^{l} & =\varepsilon^{m n l k} \gamma_{k}^{*}-\eta^{m l} \gamma^{n}+\eta^{n l} \gamma^{m}
\end{aligned}
$$

(см., например, [1] и [19], соответственно), где $\varepsilon^{\cdots}$ - символ Леви-Чивиты, ${ }_{\gamma}^{*} \gamma_{k}=$ $\frac{1}{3 !} \varepsilon_{k q r s} \gamma^{q} \gamma^{r} \gamma^{s}$. Рассмотрим два случая:

1) $l \neq m, n$, тогда (38) обрашается в нуль, но

$$
2 \Sigma^{m n} \gamma^{l} C=\varepsilon^{m n l k} \gamma_{k}^{*} C,
$$

т.к. матрицы ${ }_{\gamma}^{*} C$ антисимметричны [19], то в рассматриваемом случае больше нечего доказывать;

2 ) пусть $l=m$ (случай $l=n$ аналогичен), при $m \neq n$ из (39) имеем $2 \Sigma_{m n} \gamma^{l} C=$ $-\gamma_{n} C$, эти матрицы симметричны [19], чем молчаливо мы неоднократно пользовались, тогда (37) принимает вид тождества $-\gamma_{n} C=-\gamma_{n} C$.

Итак, может быть сформулирована следуюшая

ТеоРема 2. Фундаментальное представление группь 1 с с генераторами, удовлетворяющими соотношениям (11), существует тогда и только тогда, когда инфинитезимальное действие ее на координатном градуированном физическом пространстве $B_{c}$ может быть задано формулами

$$
\begin{aligned}
x^{\prime m} & =x^{m}+\omega^{m n} x_{n}+a^{m}+k \varepsilon^{a}\left(\theta \gamma^{m} C\right)_{a}, \\
\theta^{\prime a} & =\theta^{a}+\frac{1}{2} \omega^{m n}\left(\theta \Sigma_{m n}\right)^{a}+\frac{1}{k} \varepsilon^{a}-\rho\left(\theta i \gamma_{5}\right)^{a}, \\
r^{\prime} & =r+\rho, \quad k \in \mathbb{R} .
\end{aligned}
$$

$(10 \times 10)$-генераторами этого однопараметрического семейства действий явля- 
ются следующие: $P_{m}$ остается без изменений (см. (34)),

$$
\begin{aligned}
& J_{m n}=\left(\begin{array}{cccc}
J_{m n}^{(P)} & \vdots & \vdots & \vdots \\
\ldots \ldots \ldots & \ldots \ldots \ldots \ldots & \ldots \\
\ldots & \Sigma_{m n} & \vdots & \vdots \\
\ldots & \vdots & \vdots \\
\ldots \ldots & \ldots \ldots \ldots \ldots & \vdots \\
\ldots & \vdots & \vdots
\end{array}\right)
\end{aligned}
$$

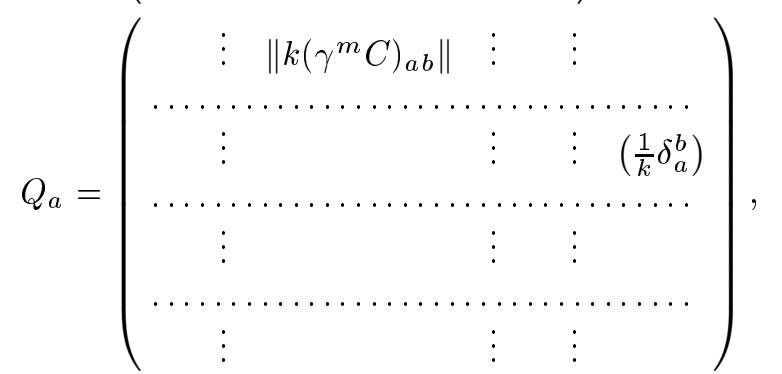

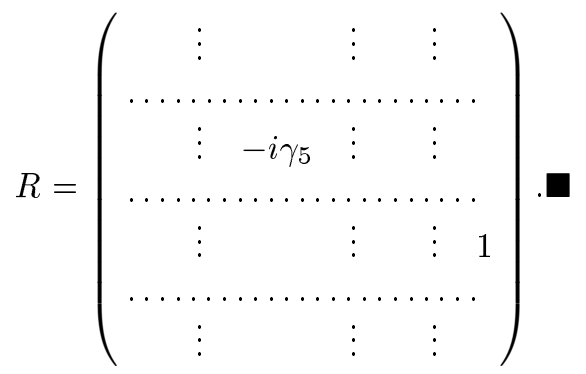

Интегральный вариант преобразования (41) имеет вид

$$
\left(\begin{array}{c}
z^{\prime} \\
1
\end{array}\right)=\exp \left\{a^{m} P_{m}+\frac{1}{2} \omega^{m n} J_{m n}+\varepsilon^{a} Q_{a}+\rho R\right\}\left(\begin{array}{c}
z \\
1
\end{array}\right)
$$

с генераторами (42).

\section{6. ОРТОЛИНЕЙНЫЙ КЛАСС ЭКВИВАЛЕНТНОСТИ СИСТЕМ КООР ДИНАТ ФИЗИЧЕСКОГО ПРОСТРАНСТВА $B$}

Покажем, что введенная аd hoc матриша (17) остается форминвариантной при преобразованиях (41), что оправдывает ее название метрики. Координаты, в которых метрика имеет вид (17), будем называть ортолинейными (аналоги галилеевых или лоренцевых в специальной теории относительности). Ортолинейные системы координат образуют класс эквивалентности. Любой другой класс получается из ортолинейного применением некоторого “криволинейного" (обшего) преобразования координат. В пределах каждого класса метрика остается форминвариантной. Естественно, в разных классах метрики разные по функциональному виду, но они связаны друг с другом процедурой тензорного преобразования. Объем информации, содержашейся в градуированной группе Пуанкаре $1 P$, равносилен объему информации, содержашейся в геометрии 
базы $B$ с метрикой (17) в ортолинейных координатах (ср. с эрлангенской программой Клейна).

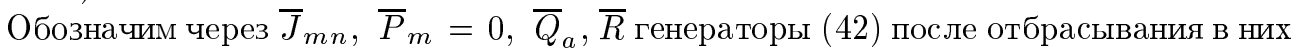
десятой строки и десятого столбца. Тогда, продифференцировав (43) по $z$, получим

$$
d z^{\prime}=\exp \left(\frac{1}{2} \omega^{m n} \bar{J}_{m n}+\varepsilon^{a} \bar{Q}_{a}+\rho \bar{R}\right) d z .
$$

ОПРЕДЕЛЕниЕ 3. Скалярная (инвариантная) величина

$$
(d s)^{2}=(d z)^{\mathrm{gt}} \eta d z=d z^{M} \eta_{M N} d z^{N}
$$

с метрикой (17) называется квадратом девятимерного градуированного интервала (или просто интервалом) пространства $B_{c}$ в ортолинейных координатах.

ОПРЕДЕЛЕНИЕ 4. Преобразование координат $\varphi_{A^{\prime}} \circ \varphi_{A}^{-1}: \varphi_{A} U_{A} \rightarrow \varphi_{A^{\prime}} U_{A^{\prime}}$, где $\varphi_{A}: U_{A} \rightarrow B_{c}, U_{A} \subset B, A \in \mathcal{A},-$ координатные диффеоморфизмы базового физического пространства $B$, оставляюшее метрику в любом классе форминвариантной, называется движением $B_{c}$.

ТЕОРема 3. Если градуированное транспонирование определить формулой (4), то преобразование (41) является движением $B_{c}$. При $\rho=0$ утверждение справедливо безусловно.

ДОКАЗАТЕЛЬСТВО проведем относительно подробно в силу нестандартности приемов и нетривиальности учета знаков. Начинаем просто: нужно убедиться в выполнимости импликации $(d s)^{2}=\left(d s^{\prime}\right)^{2} \Rightarrow \eta_{M N}=\eta_{M N}^{\prime}$ при условии (44). Штрих относится к преобразованным координатам. Имеем

$$
\begin{aligned}
(d s)^{2}= & \left(d z^{\prime}\right)^{\mathrm{gt}} \exp \left(-\frac{1}{2} \omega^{m n} \bar{J}_{m n}^{\mathrm{gt}}+\bar{Q}_{a}^{\mathrm{gt}} \varepsilon^{a}-\rho \bar{R}^{\mathrm{gt}}\right) \eta \times \\
& \times \exp \left(-\frac{1}{2} \omega^{m n} \bar{J}_{m n}-\varepsilon^{a} \bar{Q}_{a}-\rho \bar{R}\right) d z^{\prime}=\left(d s^{\prime}\right)^{2} .
\end{aligned}
$$

В случае инфинитезимальных параметров преобразования это соотношение можно представить в виде

$$
(d s)^{2}=\left(d z^{\prime}\right)^{\mathrm{gt}} \eta d z^{\prime}+\Delta(d s)^{2}=\left(d s^{\prime}\right)^{2},
$$

где

$$
\begin{aligned}
\Delta(d s)^{2}= & -\left(d z^{\prime}\right)^{\mathrm{gt}}\left(\frac{1}{2} \omega^{m n} \bar{J}_{m n}^{\mathrm{gt}}+\varepsilon^{a} \bar{Q}_{a}^{\mathrm{gt}}+\rho \bar{R}^{\mathrm{gt}}\right) \eta d z^{\prime}- \\
& -\left(d z^{\prime}\right)^{\mathrm{gt}} \eta\left(\frac{1}{2} \omega^{m n} \bar{J}_{m n}+\varepsilon^{a} \bar{Q}_{a}+\rho \bar{R}\right) d z^{\prime}
\end{aligned}
$$

По определению $\left(d z^{\prime}\right)^{\mathrm{gt}}=\left(d z^{\prime}\right)^{t}$, где индекс $t$ означает обычное транспонирование, при котором столбец преврашается в строку и наоборот.

Покажем, что в условиях теоремы $\Delta(d s)^{2}=0$. Тогда из (47) будет следовать доказываемое равенство $\eta=\eta^{\prime}$. 
Согласно (17) и (42а) в градуированной матрице $\bar{J}_{m n}^{\mathrm{gt}} \eta+\eta \bar{J}_{m n}$ векторный и спинорный диагональные блоки имеют вид соответственно

$$
\left(J_{m n}^{(P)}\right)^{t} \eta_{v}+\eta_{v} J_{m n}^{(P)} \quad \text { и } \Sigma_{m n}^{\mathrm{gt}} C+C \Sigma_{m n}
$$

По формуле (38) первый блок обрашается в нуль. Второй блок - симметричная в обычном смысле матрица относительно спинорных индексов:

$$
-\Sigma_{m n}^{t} C+C \Sigma_{m n}=\left(C \Sigma_{m n}\right)^{t}+C \Sigma_{m n}=2 C \Sigma_{m n}
$$

(знак минус возник за счет формулы (4) для матрицы $M \rightarrow \bar{J}_{m n}$ ). Поэтому двусторонняя свертка $d z^{\prime a}\left(2 C \Sigma_{m n}\right) a b d z^{\prime b}$ его с антикоммутирующими дифференциалами $d z^{\prime a}$ и $d z^{\prime b}$ исчезает. В итоге члены с $\omega^{m n}$ в $(48)$ не дают вклада.

Далее, часть

$$
\begin{aligned}
& -\left(d z^{\prime}\right)^{\mathrm{gt}}\left(\varepsilon^{a} \bar{Q}_{a}^{\mathrm{gt}} \eta+\varepsilon^{a} \eta \bar{Q}_{a}\right) d z^{\prime}= \\
& \quad=\varepsilon^{a}\left[d z^{\prime b} k\left(\gamma^{m} C\right)_{a b} \eta_{m n} d z^{\prime n}-d z_{m}^{\prime} k\left(\gamma^{m} C\right)_{a b} d z^{\prime b}\right]
\end{aligned}
$$

величины (48) также исчезает, т.к. $\varepsilon^{a}$ и $d z^{\prime b}$ антикоммутируют при всех $a, b=\overline{1,4}$. Наконец,

$$
\bar{R}^{\mathrm{gt}} \eta+\eta R=i \gamma_{5}^{t} C-i C \gamma_{5}=-i\left(C \gamma_{5}\right)^{t}-i C \gamma_{5}=0
$$

в силу антисимметричности $C \gamma_{5}$ (после первого знака равенства в (52) речь идет о спинорном диагональном блоке $(9 \times 9)$-матрицы, в которой остальные блоки тождественно нулевые). Следовательно, (48) исчезает тождественно.

При всяком ином определении градуированного транспонирования $[13,14]$ таком, что спинорный диагональный блок матрищы транспонируется обычным образом, мы получили бы $\Sigma_{m n}^{t} C+C \Sigma_{m n}=0$ (по сушеству, это не дает ничего нового в смысле вывода о том, что члены с $\omega^{m n}$ в (48) не дают вклада), а вместо (52) - выражение

$$
-i \gamma_{5}^{t} C-i C \gamma_{5}=-2 i C \gamma_{5} \neq 0
$$

(точнее, отличен от нуля лишш спинорный диагональный блок $\left(-2 i C \gamma_{5}\right)$ в $(9 \times 9)$-матрице). Антисимметричность матрищы $C \gamma_{5}$ означает, что вклад членов с генератором $\bar{R}$ в (48) исчезает лишь при $\rho=0$. Что касается $(51)$, то возможные изменения знаков при транспонировании $\bar{Q}_{a}$ и $d z^{\prime}$ обычно не изменяют результата.

В смысле определения (4) метрика (17) симметрична: $\eta=\eta^{\mathrm{gt}}$.

\section{7. РЕШЕНИЕ ПРОБЛЕМЫ СКРУЧЕННОСТИ $G$-РАССЛОЕНИЙ}

Всевозможные локальные системы координат $\varphi_{A}: U_{A} \rightarrow B_{c}, A \in \mathcal{A}$, базового физического пространства $B, U_{A} \subset B$, в которых сохраняется четность измерений, объединим в класс $C_{a r b}^{\text {ev }}$. Это означает, что в нем первые четыре измерения остаются 4-векторными (в классическом смысле), значит, четными, следуюшие четыре - спинорными (нечетными), последнее измерение - скалярным (четным). В качестве индексов будем использовать либо латинские буквы, либо их греческие аналоги в зависимости 
от того, по представлению какой янг-миллсовой группы $-L_{\mathrm{Y}-\mathrm{M}}$ или $G L_{\mathrm{Y}-\mathrm{M}}(B)-$ преобразуется индексируемая величина. Так, в сечении

$$
\sigma_{1 P}(b)=\exp \left[a^{m}(b) P_{m}+\frac{1}{2} \omega^{m n}(b) J_{m n}+\varepsilon^{a}(b) Q_{a}+\rho(b) R\right] \in 1 P_{\mathrm{Y}-\mathrm{M}}
$$

главного $1 P$-расслоения над базой $B$ функции $a^{m}(b)$ и т.д. имеют латинскую индексацию $(m, m n, a, \varnothing)$. С другой стороны, координаты (образа)

$$
z(b)=z=\left(z^{\Pi}\right)=\left(x^{\mu}, \theta^{\alpha}, r=z^{\varnothing}\right) \in B_{c}, \quad \Pi=(\mu, \alpha, \varnothing),
$$

точки $b \in B$ в произвольной локальной системе координат $\varphi_{A} \in C_{a r b}^{\mathrm{ev}}$ индексируются греческими буквами (для $x$ - из середины алфавита, $\mu=\overline{0,3}$; для $\theta$ - из начала алфавита, $\alpha=\overline{1,4})$. Это согласуется с тем, что касательные векторы к базе $B$ преобразуются по 9-векторному (в градуированном смысле) представлению группы $G L(B)$.

Для перехода от греческой к латинской индексации и наоборот нужен некоторый $б u$ тензор $E_{\Pi}^{M}(z)$, который мы назовем трансформационной связъю (в английской литературе он носит название vielbein), и обратный к нему $E_{M}^{-1 \Pi}(z)$ (символ обращения $(-1)$ часто опускают). Оба преобразуются взаимно контраградиентно по векторному и ковекторному представлениям градуированной группы $L_{\mathrm{Y}-\mathrm{M}} \times G L_{\mathrm{Y}-\mathrm{M}}(B)$ с индексацией прямого произведения. В теории дифференциальной связности $\nabla$ применительно к гравитации существует особая - метрическая - процедура выбора $E_{\Pi}^{M}(z)$, которая определяет функции связи, начальные, но важные, сведения о которых даны в [8]. Детали полнее обсудим в следующей статье.

Пусть $z_{(L)}^{M}(b)$ и $z^{(\Pi)}(b)$ - ортолинейнье и криволинейнье координаты одной и той же точки $b \in B$ и

$$
z^{\prime \Pi}(b)=z^{\Pi}(b)+\xi^{\Pi}(z),
$$

где $\xi^{\Pi}(z)=\xi_{(L)}^{M}(z) E_{M}^{\Pi}(z)$ - произвольное инфинитезимальное преобразование координат из класса $C_{a r b}^{\mathrm{ev}}$. Величину $\xi_{(L)}^{M}(z)$ будем называть полноприводимым инфинитезимальным векторным полем: его компоненты $\left(\xi_{(L)}^{m}, \xi_{(L)}^{a}, \xi_{(L)}^{\varnothing}=\rho(z)\right)$ в ортолинейных координатах преобразуются по 4-векторному, спинорному и скалярному представлениям группы $L_{\mathrm{Y}-\mathrm{M}}$. Весьма широкий подкласс $\bar{C}_{a r b}^{\mathrm{ev}}$ преобразований (55) может быть образован действием различных сечений $(53)$ на точки $z_{(L)}^{M}(b)$ по схеме $(43)$ :

$$
\left(\begin{array}{c}
z_{(L)}(b)+\xi_{(L)}(b) \\
1
\end{array}\right)=\sigma_{1 P}(b)\left(\begin{array}{c}
z_{(L)}(b) \\
1
\end{array}\right) .
$$

Инфинитезимальный полноприводимый вектор имеет компоненты

$$
\left(\begin{array}{c}
\xi_{(L)}^{m}(z) \\
\xi_{(L)}^{a}(z) \\
\xi_{(L)}^{\varnothing}(z)
\end{array}\right)=\left(\begin{array}{c}
\omega^{m n}(z) x_{(L) n}+a^{m}(z)+k \varepsilon^{a}(z)\left(\theta_{(L)} \gamma^{m} C\right)_{a} \\
\frac{1}{2} \omega^{m n}(z)\left(\theta_{(L)} \Sigma_{m n}\right)^{a}+\frac{1}{k} \varepsilon^{a}(z)-\rho(z)\left(\theta_{(L)} i \gamma_{5}\right)^{a} \\
\rho(z)
\end{array}\right)
$$

По-прежнему $k \in \mathbb{R}$. Для того чтобы числа независимых параметрических функций в левом и правом столбцах (56) совпадали и равнялись девяти, на функции $\omega^{m n}(z)$ необходимо наложить какое-то ограничение. Легко видеть, что априорное ограничение

$$
\omega^{m n}(z)=\frac{1}{2}\left(\frac{\partial \xi^{\Pi}}{\partial z_{\Lambda}}-(-1)^{\Lambda \Pi} \frac{\partial \xi^{\Lambda}}{\partial z_{\Pi}}\right) E_{\Pi}^{m}(z) E_{\Lambda}^{n}(z)
$$


обладает следующими естественными (ожидаемыми) свойствами: а) в частном случае однородных параметров преобразований в отсутствие гравитации оно согласуется с формулами (41); б) выражение (57) является тензорным в общековариантном смысле (аналог $F_{\mu \nu}=\partial_{\mu} A_{\nu}-\partial_{\nu} A_{\mu}$ в электродинамике). Знак четности $\left(-1^{\Lambda \Pi}\right)$ определяется, как в равенстве $(3)$.

Для задания функций перехода, реализуюших решение проблемы скрученности (см. раздел 2), сосредоточим внимание на отдельных прямых сомножителях группы (1). В том, что касается группы $G L(B)$, будем придерживаться концепций обычной дифференциальной геометрии или теории касательных расслоений. Используя греческую индексацию согласно (55), функции перехода с градуированными значениями в $G L_{\mathrm{Y}-\mathrm{M}}(B)$ зададим формулой

$$
\left.g_{A A^{\prime}}(z)\right|_{G L(B)}=g_{G L(B)}(z)=\left(\frac{\partial z^{\prime \Pi}}{\partial z^{\Lambda}}\right)=\left.\left(\delta \frac{\Pi}{\Lambda}+\frac{\partial \xi^{\Pi}}{\partial z^{\Lambda}}\right) \in G L_{\mathrm{Y}-\mathrm{M}}(B)\right|_{U_{A} \cap U_{A^{\prime}}}
$$

Для лоренцевых функций перехода $\left.g_{A A^{\prime}}(z)\right|_{L}$, соответствующих общим преобразованиям (55) с носителями $U_{A} \cap U_{A^{\prime}}\left(U_{A}, U_{A^{\prime}} \subset B ; A, A^{\prime} \in \mathcal{A}\right)$, можем написать очевидную полноприводимую формулу

$$
\left.g_{A A^{\prime}}(z)\right|_{L}=g_{L}(z)=\exp \left\{\frac{1}{2} \omega^{m n}(z) J_{m n}\right\} \in D_{\text {vector }}(L) \oplus D_{\text {spin }}(L)
$$

или, если имеем дело только со спинорным неприводимым представлением, формулу

$$
\left.g_{A A^{\prime}}(z)\right|_{L}=D_{\text {spin }}\left(g_{L}(z)\right)=\exp \left\{\frac{1}{2} \omega^{m n}(z) \Sigma_{m n}\right\} \in D_{\text {spin }}
$$

В (59) и (60) функции $\omega^{m n}(z)$ задаются аксиоматически формулой (57).

Наконец, функции перехода, соответствующие прямой подгруппе $G_{\text {int }}$ внутренних симметрий элементарных частиц, принимаются тождественными:

$$
\left.g_{A A^{\prime}}(z)\right|_{G_{\mathrm{int}}}=e \in G_{\mathrm{int} \mathrm{Y}-\mathrm{M}}, \quad e=e^{2}
$$

Вызвано это тем, что не видно никаких доводов в пользу их иного определения. В то же время последняя аксиома не противоречит экспериментальным фактам в физике элементарных частиц. Это следует понимать в том смысле, что, как покажем в дальнейших публикациях, развиваемый геометрический формализм $(B$-модель) удовлетворяет принципу соответствия, согласно которому $B$-модель при разрушении градуировки и в отсутствие гравитации переходит в стандартную модель Глэшоу-Вайнберга-Салама [15-17].

\section{8. ГРАДУИРОВАННЫЕ ФИЗИЧЕСКИЕ СОСТОЯНИЯ}

ОПредЕЛЕниЕ 5. Градуированным полем $\psi(z)$, описываюшим физическое состояние элементарных частиц, называется сечение такого градуированного расслоения $\zeta$ со 
структурной группой (1), в каждом слое которого действует группа (1) согласно формуле

$$
\begin{aligned}
\psi^{\prime}\left(z^{\prime}\right) & =D_{G L(B)} \times D_{L} \times D_{\text {int }}(g(z)) \psi(z)= \\
& =D(g(z)) \psi(z)=U\left(g_{G L(B)} \times g_{L} \times g_{\text {int }}\right)(z) \psi\left(z^{\prime}\right),
\end{aligned}
$$

здесь $\left.g(z) \in G_{\mathrm{Y}-\mathrm{M}}\right|_{U_{A}}, U_{A} \subset B$. Прямые сомножители $g(z)$ - также янг-миллсовы элементы. Элемент $g_{L}(z) \in L_{\mathrm{Y}-\mathrm{M}}$ либо индуцируется преобразованием (55) по формуле (57), либо берется произвольным. В трансформационном анализе первый вариант используется для вычисления величин типа момента импульса и получения соответствуюших тождеств и законов сохранения, второй - для учета калибровочной симметрии интеграла действия и получения аналогичных тождеств (ср. с результатами статьи [8]). Неприводимые представления $D_{i}, i=G L(B), L$, int, подгрупп $i$ требуют подходящих индексаций, которые мы не указываем, но подразумеваем. Оператор $U(g(z))=U(g)(z)$ преобразования поля $\psi$ вводится для удобства.

Запишем преобразование координат (55) условно в виде $z^{\prime}=g_{\xi} z,\left.g_{\xi} \in C_{a r b}^{\mathrm{ev}}\right|_{U_{A} \cap U_{A^{\prime}}}$. Тогда (62) можно переписать в виде

$$
\psi^{\prime}(z)=D(g) \psi\left(g_{\xi}^{-1} z\right)=U(g) \psi(z)
$$

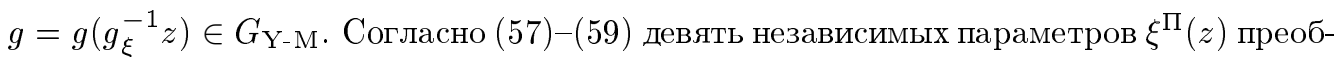
разования (55) "вторгаются" в систему параметризации групп $G L_{\mathrm{Y}-\mathrm{M}}(B)$ и $L_{\mathrm{Y}-\mathrm{M}}$, которые, таким образом, оказываются в известном смысле взаимосвязанными (впрочем, см. вьшеприведенное замечание относительно элементов $\left.g_{L}(z)\right)$. Однако в своих представлениях эти группы действуют, конечно, независимо.

Пусть $\theta^{\alpha}$ - спинорные (антикоммутируюшие) переменные в наборе $z=\left(z^{\Pi}\right)=$ $\left(x^{\mu}, \theta^{\alpha}, z^{\varnothing}=r\right)$ аргументов градуированного поля $\psi(z)$. Тогда оно может быть разложено по $\theta$ в ряд Тейлора

$$
\psi(z)=\sum_{n=0}^{4} \frac{1}{n !} \psi_{n}(z),
$$

которьй обрывается автоматически на пятом члене. Здесь $\psi_{0}(z)=\psi^{(0)}(x, r), \psi_{1}(z)=$ $\psi_{\alpha}^{(1)}(x, r) \theta^{\alpha}$ и т.д., вообще

$$
\psi_{n}(z)=\psi_{\alpha_{n} \ldots \alpha_{1}}^{(n)}(x, r) \theta^{\alpha_{1}} \ldots \theta^{\alpha_{n}}, \quad n=\overline{0,4} .
$$

В динамической теории каждьй член разложения (64) описывает соответствуюшее поколение (семейство) элементарных частиц. Таким образом, существует не более пяти поколений, описываемых унифицированно в рамках единой функции $\psi(z)$. В настоящее время экспериментально известно сушествование трех поколений элементарных частиц. Заметим, что из пяти наборов коэфффициентов функций $\psi_{\alpha_{n} \ldots \alpha_{1}}^{(n)}(x, r)$ три набора играют роль фермионных источников калибровочных полей, а два набора - бозонных (при условии, что полное поле (64) фермионное). Это различие еще предстоит исследовать теоретически. Потенциально здесь возможно действие некоторого правила запрета, в силу которого наблюдаемы не пять поколений, а только три.

2 Теоретическая и математическая физика, т. 108, № 1, 1996 г. 


\section{9. ЗАКЛЮЧЕНИЕ}

Мы убедились, что генераторы действия градуированной группы Пуанкаре $1 P$ на своей инвариантной подгруппе $1 T$ не удовлетворяют исходным коммутационным соотношениям (11). Следовательно, в отличие от классического варианта, это действие не является представлением группы. Эвристический поиск адекватных инфинитезимальных преобразований ортолинейных координат в физическом пространстве $B_{c}$ увенчался формулами (41) и (42). Оказалось, что они порождают однопараметрическое семейство генераторов, совместимых с соотношениями (11). Роль свободного параметра $k \in \mathbb{R}$ этого семейства еше не выяснена.

Общекоординатные преобразования (55) влекут за собой конструирование янг-миллсовой группы $G L_{\mathrm{Y}-\mathrm{M}}(B)(58)$, действуюшей в расслоениях касательных тензоров над девятимерным базовым градуированным физическим пространством $B$ с подстилаюшим линейным координатным пространством $B_{c}$. Эти же преобразования по формуле (57) задают параметры $\omega^{m n}(z)$ функций перехода (59) или (60) со значениями в группе $L_{\mathrm{Y}-\mathrm{M}}$ или в спинорном представлении янг-миллсовой группы Лоренца. Действием "посредников" $G L_{\mathrm{Y}-\mathrm{M}}(B)$ и $L_{\mathrm{Y}-\mathrm{M}}$ в гильбертовом пространстве $H$ физических состояний $\psi(z)$ (см. раздел 8) ограничивается действие в $H$ преобразования (55). В связи с этим необходимо сделать следующее предостережение.

В теории суперсимметрии (например, [1-6]) практикуют подмену групшы $C_{a r b}^{\text {ev }}$ обших преобразований координат и группы (1) с действием (62) группой физических операторов (токов и зарядов), действуюших в $H$ в соответствии со структурой супергруппы Пуанкаре $1 P$. Так возникает киральное неприводимое представление группы $1 P$, которое играет основную роль в теории суперсимметрии. На наш взгляд, такая подмена недопустима. Действительно, все физические операторы из класса токов и зарядов по своей природе являются вторичны.ми по отношению к группам (1) и $C_{a r b}^{\text {ev }}$. Это следует из того, что если задан интеграл действия $A$, выраженный через градуированные поля $\psi(z)$, то по схеме (62) можно выполнить трансформационный анализ в духе статьи [8] и только после этого получить сохраняющиеся величины - токи и заряды. Нельзя следствие класть в основу теории. Феноменологический подход к теории суперсимметрии, допускаюший указанную подмену, в этом смысле не оправдание. Заметим, что поля $\psi(z)$ вообше не реализуют никакого представления группы $1 P$. В теории же суперсимметрии именно эти представления принимаются во внимание, что приводит к трудностям в конструировании интеграла действия и большим проблемам относительно выполнения принципа соответствия (см. [1-6]).

Важным геометрическим результатом является доказательство того, что градуированная матрица (17) выполняет роль метрического тензора над базовым физическим пространством $B$ в ортолинейных координатах. В первую очередь этот результат необходим при корректном конструировании интеграла действия для элементарных частиц и гравитации. Следует, однако, подчеркнуть, что такое конструирование должно быть осушествлено с привлечением новых нетривиальных математических приемов, о чем речь пойдет в следующей статье.

Легко понять, что аксиомы в разделе 7 обеспечивают ковариантное описание спина. Если их использовать в полном трансформационном анализе, то получаются не только 
ожидаемые $[8]^{1)}$ законы сохранения (за счет функций $\xi^{\mu}(z)$ ), но и новые (за счет функций $\xi^{\alpha}(z)$ и $\left.\xi^{\varnothing}(z)\right)$. На этом пути можно получить глубокие физические результаты. Ясно, что последнее - предмет отдельных работ.

\section{Список литературы}

[1] П. Уэст. Введение в суперсимметрию и супергравитацию. М.: Мир, 1989.

[2] Ю. Весс, Дж. Беггер. Суперсимметрия и супергравитация. М.: Мир, 1986.

[3] M. F. Sohnius. Introducing Supersymmetry // Phys. Rep. (Review Section of Physics Letters). Amsterdam: North-Holland, 1985. V. 128. № 2, 3. P. 39-204.

[4] D. Z. Freedman. Introduction to Supersymmetry in Particle and Nuclear Physics. New York: Plenum Press, 1984. P. 1-28.

[5] M. Müller. Consistent Classical Supergravity Theories. Lecture Notes in Physics (№ 336). Berlin: Springer-Verlag, 1989.

[6] B. de Witt, D. Z. Freedman. Supersymmetry. New York: Plenum Press, 1985. P. 135-210.

[7] R. Haag, J. Lopuszanski, M. Sohnius // Nucl. Phys. 1967. V. B88. P. 257.

[8] В. Т. Березин // ТМФ. 1992. Т. 93. № 1. С. 154-174.

[9] К. Годбийон. Дифференциальная геометрия и аналитическая механика. М.: Мир, 1973.

[10] Д. Хьюзмоллер. Расслоенные пространства. М.: Мир, 1970.

[11] М. М. Постников. Дифференциальная геометрия. М.: Наука, 1988.

[12] Р. Зуланке, П. Винтген. Дифференциальная геометрия и расслоения. М.: Мир, 1975.

[13] B. de Witt. The Spacetime Approach to Quantum Field Theory // Course 5. Relativity, groups and topology. II / Eds. B.S. de Witt and R. Stora. Les Houches, Session XL, 1983. Elsevier Sc. Publ., 1984. P. 482-738.

[14] $\Phi . А$. Березин. Введение в алгебру и анализ с антикоммутирующими переменными. М.: Изд-во МГУ, 1983.

[15] Г. Кейн. Современная физика элементарных частиц. М.: Мир, 1990.

[16] Т.-П. Ченг, Л.-Ф. Ли. Калибровочные теории в физике элементарных частиц. М.: Мир, 1987.

[17] Л. Райдер. Квантовая теория поля. М.: Мир, 1987.

[18] М. М. Постников. Группы и алгебры Ли. М.: Наука, 1982.

[19] В. А. ЖКелнорович. Теория спиноров и ее применение в физике и механике. М.: Наука, 1982.

Донецкий физико-технический институт

им. А. А. Галкина НАН Украины

Поступила в редакцию 16.VIII.1995 г

\section{V.T. Berezin \\ GEOMETRICAL STRUCTURE OF THE BASE AND TWISTING OF THE GRADED FIBRE BUNDLES USED IN MODELING GRAVITATION AND ELEMENTARY PARTICLES}

To deseribe the unification of the fundamental interactions of elementary particles and gravitation the graded bundle mathematical structure $\zeta$ is needed. Its base $B$ is the 9 -dimensional graded space having one scalar, four spinor and four vector dimensions. One-parametric family of the Poincaré group $1 P$ is found. It is shown that any group of this family acts on its invariant subgroup and on the base $B$ in different ways. This situation is different from the classical one and point out at the nontriviality of the $\zeta$-bundle geometrical properties. The problem of twisting is discussed.

\footnotetext{
${ }^{1)}$ В статье [8] имеются опечатки. В частности, в формуле $(22)$ вместо $\cdots=\left[\cdots+\frac{\delta L_{s}}{\delta \varepsilon_{\mu \nu}}\right]_{\varepsilon \in H}$ должно быть $\cdots=\left[\cdots+\frac{\delta L}{\delta \varepsilon_{\mu \nu}}\right]_{\varepsilon \in H}$.
} 